MATHEMATICS OF COMPUTATION

Volume 81, Number 277, January 2012, Pages 585-603

S $0025-5718(2011) 02473-7$

Article electronically published on June 21, 2011

\title{
UNIVERSAL CYCLES FOR MINIMUM COVERINGS OF PAIRS BY TRIPLES, WITH APPLICATION TO 2-RADIUS SEQUENCES
}

\author{
YEOW MENG CHEE, SAN LING, YIN TAN, AND XIANDE ZHANG
}

\begin{abstract}
A new ordering, extending the notion of universal cycles of Chung et al. (1992), is proposed for the blocks of $k$-uniform set systems. Existence of minimum coverings of pairs by triples that possess such an ordering is established for all orders. The application to the construction of short 2-radius sequences is given, along with some new 2-radius sequences found through a computer search.
\end{abstract}

\section{INTRODUCTION}

Determining the existence of orderings on the blocks of various classes of set systems (all terms are defined in the next section) to meet specified criteria is a fundamental problem in discrete mathematics, due to applications in combinatorial computing. The following two types of orderings have long histories:

(i) An ordering of the blocks such that any two successive blocks have symmetric difference of the smallest possible size (the size of one for set systems having more than one block size, and the size of two for $k$-uniform set systems). This type of ordering was first studied by Gray [16] and has now come to be known as combinatorial Gray codes 27. Combinatorial Gray codes are known to exist for the complete set system $\left(X, 2^{X}\right)$ [16, for the complete $k$-uniform set system $\left(X,\left(\begin{array}{l}X \\ k\end{array}\right)\right)[3,11,26$, and for some classes of triple systems with index two 10 .

(ii) An ordering of the blocks such that any two successive blocks have nonempty intersection. Such an ordering is also equivalent to a hamiltonian path (or hamiltonian cycle, if one also insists that the first block and last block have nonempty intersection) in the block intersection graph of the set system. The existence question for this type of ordering for Steiner triple systems was first raised by Ron Graham in 1987 at an American Mathematical Society meeting. It is known that such orderings exist for Steiner 2-designs [20, for pairwise balanced designs whose maximum block size is at most twice the minimum block size [2, for pairwise balanced

Received by the editor September 20, 2009 and, in revised form, August 6, 2010.

2010 Mathematics Subject Classification. Primary 05B05, 05B07, 05B40; Secondary 05C38, $68 \mathrm{R} 05$.

Key words and phrases. Alternating Hamiltonian cycle, block intersection graph, group divisible design, minimum covering, sequence of radius two, Steiner triple system, universal cycle.

This research was supported in part by the National Research Foundation of Singapore under Research Grant NRF-CRP2-2007-03 and by the Nanyang Technological University under Research Grant M58110040. 
designs whose minimum block size is at least three [18, and for triple systems of arbitrary index [19.

Recently, Dewar [10] studied universal cycles for block designs. This is an ordering of the blocks such that two successive blocks differ in a small structural way. The concept of universal cycles was introduced by Chung et al. [6] as a generalization of de Bruijn sequences [9. Universal cycles are known to exist for the complete $k$-uniform set system $\left(X,\left(\begin{array}{l}X \\ k\end{array}\right)\right)$ when $k \in\{2,3,4,5,6\}$, provided $k$ divides $\left(\begin{array}{c}|X|-1 \\ k-1\end{array}\right)$ and $|X|$ is sufficiently large [6, 23, 21, 22, and for some classes of triple systems of index two [10].

Under the definition of universal cycles of Chung et al. [6], universal cycles cannot exist for $k$-uniform set systems in which there are two blocks intersecting in less than $k-1$ points. To overcome this restriction, Dewar [10 proposed an extension to the definition of universal cycles and showed that with this new definition there exist universal cycles for some classes of Steiner 2-designs, including Steiner triple systems. However, these new universal cycles of Dewar have the disadvantage that the blocks of a set system cannot always be recovered from its universal cycle.

In this paper, the concept of $s$-shift universal cycles is proposed as another natural extension to the universal cycles of Chung et al. 6]. Minimum $(n, 3,2)$ coverings for which there exist 2-shift universal cycles are constructed for all $n \geq 3$ by considering alternating hamiltonian cycles in their block intersection graphs. An application to the construction of 2-radius sequences of order $n$ [24] is given.

\section{Notation, TERMinOlOGy, AND KNOWN RESUlts}

The ring $\mathbb{Z} / m \mathbb{Z}$ is denoted by $\mathbb{Z}_{m}$, and the set of nonnegative integers is denoted by $\mathbb{Z}_{\geq 0}$. For $n$ a positive integer, the set $\{1,2, \ldots, n\}$ is denoted by $[n]$. For $X$ a finite set and $k$ an integer, $0 \leq k \leq|X|$, the set of all $k$-subsets of $X$ is denoted by $\left(\begin{array}{l}X \\ k\end{array}\right)$.

A set system is a pair $\mathfrak{S}=(X, \mathcal{A})$, where $X$ is a finite set and $\mathcal{A} \subseteq 2^{X}$. The members of $X$ are called vertices or points, and the members of $\mathcal{A}$ are called edges or blocks. The order of $\mathfrak{S}$ is the number of vertices $|X|$, and the size of $\mathfrak{S}$ is the number of edges $|\mathcal{A}|$. Let $K \subseteq \mathbb{Z}_{\geq 0}$. The set system $\mathfrak{S}$ is said to be $K$-uniform if $|A| \in K$ for all $A \in \mathcal{A}$. We often write $\{k\}$-uniformity as $k$-uniformity. A 2-uniform set system is also known simply as a graph.

An edge-colored graph is a pair $(\Gamma, \chi)$, where $\Gamma=(X, \mathcal{A})$ is a graph and $\chi$ is a function on $\mathcal{A}$ (called a coloring). The image of $\chi, \mathrm{C}=\{\chi(A): A \in \mathcal{A}\}$, is called the color set. Elements of the color set are called colors. An edge $A \in \mathcal{A}$ is said to have color $c \in \mathrm{C}$ if $\chi(A)=c$. A cycle in an edge-colored graph is colorful if for every color there exists some edge in the cycle having that color. An alternating cycle in an edge-colored graph is a cycle where adjacent edges have distinct colors.

A pairwise balanced design (PBD), or more specifically a $\operatorname{PBD}(n, K)$, is a $K$ uniform set system $(X, \mathcal{A})$ of order $n$ such that every $T \in\left(\begin{array}{c}X \\ 2\end{array}\right)$ is contained in exactly one block of $\mathcal{A} \cdot \operatorname{A} \operatorname{PBD}(n,\{3\})$ is called a Steiner triple system of order $n$ and is denoted by $\operatorname{STS}(n)$. It is well known (see, for example, 8 ) that an $\operatorname{STS}(n)$ exists if and only if $n \equiv 1$ or $3(\bmod 6)$.

A covering of pairs by triples of order $n$ (or $(n, 3,2)$-covering) is a 3 -uniform set system $(X, \mathcal{A})$ of order $n$ such that every $T \in\left(\begin{array}{c}X \\ 2\end{array}\right)$ is contained in at least one block of $\mathcal{A}$. The minimum size of an $(n, 3,2)$-covering is the covering number $C(n, 3,2)$. An $(n, 3,2)$-covering of size $C(n, 3,2)$ is said to be minimum. Fort and Hedlund 
[12] determined that for all $n \geq 3$,

$$
C(n, 3,2)=\left\lceil\frac{n}{3}\left\lceil\frac{n-1}{2}\right\rceil\right\rceil .
$$

Note that an $\operatorname{STS}(n)$ is a minimum $(n, 3,2)$-covering.

Let $(X, \mathcal{A})$ be a set system, and let $\mathcal{G}=\left\{G_{1}, G_{2}, \ldots, G_{s}\right\}$ be a partition of $X$ into subsets, called groups. The triple $(X, \mathcal{G}, \mathcal{A})$ is a group divisible design (GDD) when every 2-subset of $X$ not contained in a group appears in exactly one block, and $|A \cap G| \leq 1$ for all $A \in \mathcal{A}$ and $G \in \mathcal{G}$. We denote a GDD $(X, \mathcal{G}, \mathcal{A})$ by $K$-GDD if $(X, \mathcal{A})$ is $K$-uniform. The type of a $\operatorname{GDD}(X, \mathcal{G}, \mathcal{A})$ is the multiset $[|G|: G \in \mathcal{G}]$. When more convenient, the exponentiation notation is used to describe the type of GDD: a GDD of type $g_{1}^{t_{1}} g_{2}^{t_{2}} \ldots g_{s}^{t_{s}}$ is a GDD where there are exactly $t_{i}$ groups of size $g_{i}, i \in[s] . \mathrm{A} \operatorname{PBD}(n, K)$ can be regarded as a $K$-GDD of type $1^{n}$, where each group contains a single point.

The existence of classes of PBDs and GDDs required in this paper is given below.

Theorem 2.1 (Gronau, Mullin, and Pietsch [17]). There exists a $\operatorname{PBD}(n,\{3$, $4,5,6,8\})$, for all $n \geq 3$.

Theorem 2.2 (Lenz [25]). There exists a $\operatorname{PBD}(n,\{4,5,6,7\})$, for all $n \geq 4$, except when $n \in\{8,9,10,11,12,14,15,18,19,23\}$.

Theorem 2.3 (Colbourn, Hoffman, and Rees [7). Let $g, t, u \in \mathbb{Z}_{\geq 0}$. There exists $a\{3\}$-GDD of type $g^{t} u^{1}$ if and only if the following conditions are all satisfied:

(i) if $g>0$, then $t \geq 3$, or $t=2$ and $u=g$, or $t=1$ and $u=0$, or $t=0$;

(ii) $u \leq g(t-1)$ or $g t=0$;

(iii) $g(t-1)+u \equiv 0(\bmod 2)$ or $g t=0$;

(iv) $g t \equiv 0(\bmod 2)$ or $u=0$;

(v) $g^{2}\left(\begin{array}{l}t \\ 2\end{array}\right)+g t u \equiv 0(\bmod 3)$.

Theorem 2.4 (Brouwer, Schrijver, and Hanani [5]). There exists a $\{4\}$-GDD of type $g^{t}$ if and only if $t \geq 4$ and

(i) $g \equiv 1$ or $5(\bmod 6)$ and $t \equiv 1$ or $4(\bmod 12)$; or

(ii) $g \equiv 2$ or $4(\bmod 6)$ and $t \equiv 1(\bmod 3)$; or

(iii) $g \equiv 3(\bmod 6)$ and $t \equiv 0$ or $1(\bmod 4)$; or

(iv) $g \equiv 0(\bmod 6)$,

with the two exceptions of types $2^{4}$ and $6^{4}$, for which $\{4\}$-GDDs do not exist.

Given a set system $\mathfrak{S}=(X, \mathcal{A})$ or $\operatorname{GDD} \mathfrak{S}=(X, \mathcal{G}, \mathcal{A})$, the block intersection graph of $\mathfrak{S}$ is a loopless multigraph $\Gamma_{\mathfrak{S}}=(Y, \mathcal{B})$ such that $Y=\mathcal{A}$, and there exist $\lambda$ edges between distinct $A, A^{\prime} \in Y$ if and only if $\left|A \cap A^{\prime}\right|=\lambda$. Let $\chi$ be a function that assigns to each edge between $A, A^{\prime} \in Y$ a distinct color in $A \cap A^{\prime}$. Then $\left(\Gamma_{\mathfrak{S}}, \chi\right)$ is called the edge-colored block intersection graph of $\mathfrak{S}$. A set system or GDD is colorful alternating hamiltonian (c.a.h.) if its edge-colored block intersection graph has a c.a.h. cycle. For brevity, a c.a.h. cycle in the edge-colored block intersection graph of a set system or GDD is simply referred to as a c.a.h. cycle in the set system or GDD.

\section{3. $s$-SHIFT UNIVERSAL CYCLES}

Let $\mathcal{F}$ be a set of combinatorial objects, each of "rank" $r$, such that each $F \in \mathcal{F}$ is specified by a sequence $\left\langle x_{1}, x_{2}, \ldots, x_{r}\right\rangle$, where $x_{i} \in X$, for some fixed $X$. 
Definition 3.1 (Universal cycle [6]). $U=\left(u_{0}, u_{1}, \ldots, u_{|\mathcal{F}|-1}\right)$ is a universal cycle for $\mathcal{F}$ if $\left\langle u_{i+1}, u_{i+2}, \ldots, u_{i+r}\right\rangle, i \in \mathbb{Z}_{|\mathcal{F}|}$, runs through each element of $\mathcal{F}$ exactly once.

Given a $k$-uniform set system $\mathfrak{S}=(X, \mathcal{A})$, it is natural to ask if there exists a universal cycle for $\mathfrak{S}$, that is, a cycle $\left(x_{0}, x_{1}, \ldots, x_{|\mathcal{A}|-1}\right)$ such that $\left\{x_{i+1}, x_{i+2}, \ldots\right.$, $\left.x_{i+k}\right\}$ runs through $\mathcal{A}$ exactly once, for $0 \leq i<|\mathcal{A}|$. Notice that for $0 \leq i<j<|\mathcal{A}|$, $\left\{x_{i+1}, x_{i+2}, \ldots, x_{i+k}\right\}$ and $\left\{x_{j+1}, x_{j+2}, \ldots, x_{j+k}\right\}$ intersect in $k-(j-i)$ points. In particular, for every $i \in\{0,1, \ldots, k-1\}, \mathcal{A}$ must contain a pair of blocks intersecting in $i$ points. This rules out the existence of universal cycles for $k$ uniform set systems in which there are no pairs of blocks that intersect in $j$ points, for some $j \in\{0,1, \ldots, k-1\}$. In particular, there cannot exist universal cycles for Steiner triple systems.

To overcome the stringent condition for the existence of universal cycles for set systems, Dewar [10] relaxed the condition by allowing a universal sequence for a $k$-uniform set system $\mathfrak{S}$ to just generate some representation of $\mathfrak{S}$ instead of generating all the blocks of $\mathfrak{S}$. The specific representation considered by Dewar is defined as follows. Let $\mathfrak{S}=(X, \mathcal{A})$ be a set system. A set $\mathcal{R} \subseteq\left(\begin{array}{l}X \\ r\end{array}\right)$ is said to represent $\mathfrak{S}$ if every block in $\mathcal{A}$ contains exactly one element of $\mathcal{R}$ and if every $R \in \mathcal{R}$ is contained in exactly one block of $\mathcal{A}$.

Example 3.2. Consider the $\operatorname{STS}(7)$ whose blocks are $\{1,3,7\},\{1,5,6\},\{4,5,7\}$, $\{2,6,7\},\{3,4,6\},\{1,2,4\}$, and $\{2,3,5\}$. This set system can be represented by $R=\{\{1,3\},\{1,5\},\{5,7\},\{6,7\},\{4,6\},\{2,4\},\{2,3\}\}$, for which there exists a universal cycle $(3,1,5,7,6,4,2)$.

One disadvantage with Dewar's approach is that the set system may not be recoverable from a given universal cycle of its representation, as seen in the example below.

Example 3.3. Consider the $\operatorname{STS}(7)$ whose blocks are $\{1,3,4\},\{2,3,6\},\{2,4,7\}$, $\{4,5,6\},\{1,6,7\},\{3,5,7\}$, and $\{1,2,5\}$. This set system is distinct from that in Example 3.2 , but it has the same representation $R=\{\{1,3\},\{1,5\},\{5,7\},\{6,7\}$, $\{4,6\},\{2,4\},\{2,3\}\}$.

Here, the notion of universal cycles is extended in another direction.

Definition 3.4 ( $s$-Shift universal cycle). Let $s$ be a positive integer. $U=\left(u_{0}\right.$, $\left.u_{1}, \ldots, u_{s|\mathcal{F}|-1}\right)$ is an $s$-shift universal cycle for $\mathcal{F}$ if $\left\langle u_{s i+1}, u_{s i+2}, \ldots, u_{s i+r}\right\rangle, i \in$ $\mathbb{Z}_{|\mathcal{F}|}$, runs through each element of $\mathcal{F}$ exactly once.

A 1-shift universal cycle is equivalent to the universal cycle of Chung et al. [6].

Example 3.5. $U=(1,3,7,2,6,4,3,5,2,1,4,7,5,6)$ is a 2 -shift universal cycle for the STS(7) in Example 3.2. The blocks of the STS(7) can be recovered from $U$.

The next result gives the equivalence between certain shift universal cycles and alternating hamiltonian cycles.

Proposition 3.6. For $k \geq 2$, a (k-1)-shift universal cycle for a $k$-uniform set system $\mathfrak{S}$ is equivalent to an alternating hamiltonian cycle in $\mathfrak{S}$.

Proof. Suppose $U=\left(u_{0}, u_{1}, \ldots, u_{m(k-1)-1}\right)$ is a $(k-1)$-shift universal cycle for a $k$-uniform set system $\mathfrak{S}$. Let $A_{i}=\left\{u_{i(k-1)}, u_{i(k-1)+1}, \ldots, u_{i(k-1)+(k-1)}\right\}, i \in \mathbb{Z}_{m}$. 
Then $\left\{A_{0}, A_{1}\right\},\left\{A_{1}, A_{2}\right\}, \ldots,\left\{A_{m-1}, A_{0}\right\}$ are edges of an alternating hamiltonian cycle in $\mathfrak{S}$, noting that the color of edge $\left\{A_{i}, A_{i+1}\right\}$ is $u_{(i+1)(k-1)}, i \in \mathbb{Z}_{m}$. Hence $\left\{A_{i}, A_{i+1}\right\}$ and $\left\{A_{i+1}, A_{i+2}\right\}$ cannot possibly be of the same color since $u_{(i+1)(k-1)}$ and $u_{(i+2)(k-1)}$ are both contained in the $A_{i+1}$.

Conversely, suppose that $\mathcal{A}=\left\{A_{0}, A_{1}, \ldots, A_{m-1}\right\}$ is the set of blocks of a $k$ uniform set system $\mathfrak{S}$ and that $\left\{A_{0}, A_{1}\right\},\left\{A_{1}, A_{2}\right\}, \ldots,\left\{A_{m-1}, A_{0}\right\}$ are edges of an alternating hamiltonian cycle in $\mathfrak{S}$. For $i \in \mathbb{Z}_{m}$, order the points within $A_{i}$ so that

(i) its first point is the color of the edge $\left\{A_{i-1}, A_{i}\right\}$ and

(ii) its last point is the color of the edge $\left\{A_{i}, A_{i+1}\right\}$.

According to this order, construct the sequence obtained by listing the points of $A_{0}, A_{1}, \ldots, A_{m-1}$, with the condition that only one of the last points of $A_{i}$ and the first point of $A_{i+1}$ is included in the listing. This sequence is a $k$-shift universal cycle for $\mathfrak{S}$.

In the next section, the existence of minimum $(n, 3,2)$-coverings that admit 2 shift universal cycles is established for all $n \geq 3$, by considering their alternating hamiltonian cycles.

\section{Alternating Hamiltonian CyCles in edge-COlored BLOCK INTERSECTION GRAPHS}

Some useful recursive constructions are first described.

4.1. Recursive constructions. We begin with a simple observation.

Proposition 4.1. Let $(\Gamma, \chi)$ be an edge-colored block intersection graph, and let $C_{1}$ and $C_{2}$ be two (vertex) disjoint alternating cycles, of length $m_{1}$ and $m_{2}$, respectively. If there is an edge in $C_{1}$ of the same color as some edge in $C_{2}$, then $\Gamma$ has an alternating cycle of length $m_{1}+m_{2}$.

Proof. Suppose $A=\{a, b\}$ and $B=\{c, d\}$ are edges in $C_{1}$ and $C_{2}$, respectively, such that $\chi(A)=\chi(B)$. The $\left(C_{1} \backslash\{a, b\}\right) \cup\left(C_{2} \backslash\{c, d\}\right) \cup\{\{a, c\},\{b, d\}\}$ is an alternating cycle of length $m_{1}+m_{2}$.

Proposition 4.2 (Filling in groups). If there exists a c.a.h. $\{3\}$-GDD of type $\left[g_{1}, g_{2}, \ldots, g_{t}\right]$, and for each $i \in[t]$ there exists a c.a.h. $\left(g_{i}, 3,2\right)$-covering, then there exists a c.a.h. $\left(\sum_{i=1}^{t} g_{i}, 3,2\right)$-covering.

Proof. Let $\mathfrak{D}=(X, \mathcal{G}, \mathcal{A})$ be a c.a.h. $\{3\}$-GDD of type $\left[g_{1}, g_{2}, \ldots, g_{t}\right]$, with $C$ as a c.a.h. cycle in $\mathfrak{D}$. For each group $G \in \mathcal{G}$, let $\mathfrak{D}_{G}=\left(G, \mathcal{B}_{G}\right)$ be a c.a.h. $(|G|, 3,2)$ covering, with $C_{G}$ as a c.a.h. cycle in $\mathfrak{D}_{G}$. It is clear that $\mathfrak{D}^{*}=\left(X, \mathcal{A} \cup\left(\bigcup_{G \in \mathcal{G}} \mathcal{B}_{G}\right)\right)$ is a $\left(\sum_{i=1}^{t} g_{i}, 3,2\right)$-covering. Each of $C_{G}$ contains an edge of the same color as some edge in $C$, so these can be combined with $C$ via Proposition 4.1 to give one c.a.h. cycle for $\mathfrak{D}^{*}$.

The following is another useful construction for c.a.h. ( $n, 3,2)$-coverings from GDDs.

Proposition 4.3 (Adjoining $y$ points and filling in groups). Let $y \in \mathbb{Z}_{\geq 0}$. Suppose there exists a (master) c.a.h. $\{3\}$-GDD of type $\left[g_{1}, g_{2}, \ldots, g_{t}\right]$, and suppose the following (ingredients) also exist:

(i) a c.a.h. $\left(g_{t}+y, 3,2\right)$-covering,

(ii) a c.a.h. $\{3\}$-GDD of type $1^{g_{i}} y^{1}$, for $i \in[t-1]$. 
Then there exists a c.a.h. $\left(y+\sum_{i=1}^{t} g_{i}, 3,2\right)$-covering.

Proof. Let $(X, \mathcal{G}, \mathcal{A})$ be a c.a.h. $\{3\}$-GDD of type $\left[g_{1}, g_{2}, \ldots, g_{t}\right]$, with $\mathcal{G}=\left\{G_{1}, G_{2}\right.$, $\left.\ldots, G_{t}\right\}$ such that $\left|G_{i}\right|=g_{i}, i \in[t]$. Let $Y$ be a set of size $y$, disjoint from $X$. Let $\left(G_{t} \cup Y, \mathcal{B}_{t}\right)$ be a c.a.h. $\left(g_{t}+y, 3,2\right)$-covering and $\left(G_{i} \cup Y,\left\{\{x\}: x \in G_{i}\right\} \cup\{Y\}, \mathcal{B}_{i}\right)$ be a c.a.h. $\{3\}$-GDD of type $1^{g_{i}} y^{1}$, for $i \in[t-1]$. Then the set system

$$
\mathfrak{S}=\left(X \cup Y, \mathcal{A} \cup\left(\bigcup_{i=1}^{t} \mathcal{B}_{i}\right)\right)
$$

is a $\left(y+\sum_{i=1}^{t} g_{i}, 3,2\right)$-covering. To show that $\mathfrak{S}$ is c.a.h., mimic the proof of Proposition 4.2

The following construction is similar to that in Proposition 4.3. except that we end up with a GDD with smaller groups instead of an $(n, 3,2)$-covering.

Proposition 4.4 (Adjoining $y$ points and breaking up groups). Let $y \in \mathbb{Z}_{\geq 0}$. Suppose there exists a (master) c.a.h. $\{3\}$-GDD of type $\left[g_{1}, g_{2}, \ldots, g_{t}\right]$, and suppose that an (ingredient) c.a.h. $\{3\}$-GDD of type $h^{g_{i} / h} y^{1}$ exists for each $i \in[t]$. Then there exists a c.a.h. $\{3\}-G D D$ of type $h^{\left(\sum_{i=1}^{t} g_{i}\right) / h} y^{1}$.

\begin{tabular}{|c|c|}
\hline Input: & $\begin{array}{l}\text { (master) GDD } \mathfrak{D}=(X, \mathcal{G}, \mathcal{A}) \\
\text { weight function } \omega: X \rightarrow \mathbb{Z}_{\geq 0} ; \\
\text { (ingredient) } K \text {-GDD } \mathfrak{D}_{A}=\left(X_{A}, \mathcal{G}_{A}, \mathcal{B}_{A}\right) \text { of type }[\omega(a): a \in A] \text {, } \\
\text { for each block } A \in \mathcal{A}, \text { where } \\
X_{A}=\bigcup_{a \in A}\{\{a\} \times\{1,2, \ldots, \omega(a)\}\} \text { and } \\
\mathcal{G}_{A}=\{\{a\} \times\{1,2, \ldots, \omega(a)\}: a \in A\} \text {. }\end{array}$ \\
\hline Output: & $\begin{array}{l}K \text {-GDD } \mathfrak{D}^{*}=\left(X^{*}, \mathcal{G}^{*}, \mathcal{A}^{*}\right) \text { of type }\left[\sum_{x \in G} \omega(x): G \in \mathcal{G}\right], \text { where } \\
X^{*}=\bigcup_{x \in X}(\{x\} \times\{1,2, \ldots, \omega(x)\}), \\
\mathcal{G}^{*}=\left\{\bigcup_{x \in G}(\{x\} \times\{1,2, \ldots, \omega(x)\}): G \in \mathcal{G}\right\}, \text { and } \\
\mathcal{A}^{*}=\bigcup_{A \in \mathcal{A}} \mathcal{B}_{A} .\end{array}$ \\
\hline & $\mathfrak{D}^{*}=\operatorname{WFC}\left(\mathfrak{D}, \omega,\left\{\mathfrak{D}_{A}: A \in \mathcal{A}\right\}\right)$ \\
\hline Note: & By convention, for $x \in X,\{x\} \times\{1,2, \ldots, \omega(x)\}=\emptyset$ if $\omega(x)=0$. \\
\hline
\end{tabular}

Figure 1. Wilson's Fundamental Construction for GDDs.

Proof. Let $(X, \mathcal{G}, \mathcal{A})$ be a c.a.h. $\{3\}$-GDD of type $\left[g_{1}, g_{2}, \ldots, g_{t}\right]$, with $\mathcal{G}=\left\{G_{1}, G_{2}\right.$, $\left.\ldots, G_{t}\right\}$. Let $Y$ be a set of size $y$, disjoint from $X$. Let $\left(G_{i} \cup Y, \mathcal{G}_{i}, \mathcal{B}_{i}\right)$ be a c.a.h. $\{3\}$-GDD of type $h^{\left|G_{i}\right| / h} y^{1}$, with $Y$ as the group of size $y$. Then the set system

$$
\mathfrak{S}=\left(X \cup Y, \bigcup_{i=1}^{t} \mathcal{G}_{i}, \mathcal{A} \cup\left(\bigcup_{i=1}^{t} \mathcal{B}_{i}\right)\right)
$$

is a $\{3\}$-GDD of type $h^{\left(\sum_{i=1}^{t} g_{i}\right) / h} y^{1}$. To show that $\mathfrak{S}$ is c.a.h., mimic the proof of Proposition 4.2 .

For Propositions 4.2, 4.3 and 4.4 to be useful, large classes of c.a.h. $\{3\}$-GDDs are needed. These can be produced with the next theorem, a direct analogue of Wilson's Fundamental Construction for GDDs 30 (shown in Figure 1), for c.a.h. GDDs. 
Theorem 4.5 (Fundamental construction). Let $\mathfrak{D}=(X, \mathcal{G}, \mathcal{A})$ be a (master) GDD, and let $\omega: X \rightarrow \mathbb{Z}_{\geq 0}$ be a weight function. Suppose that for each $A \in \mathcal{A}$, there exists an (ingredient) c.a.h. $K$-GDD $\mathfrak{D}_{A}$ of type $[\omega(a): a \in A]$. Then there exists a c.a.h. $K$-GDD $\mathfrak{D}^{*}$ of type $\left[\sum_{x \in G} \omega(x): G \in \mathcal{G}\right]$.

Proof. Apply Wilson's Fundamental Construction (Figure 1) to obtain a $K$-GDD $\mathfrak{D}^{*}$ of type $\left[\sum_{x \in G} \omega(x): G \in \mathcal{G}\right]$. That this GDD is c.a.h. can be seen as follows. Let $C_{A}$ be a c.a.h. cycle in the $K$-GDD $\mathfrak{D}_{A}, A \in \mathcal{A}$. There exists an edge in each of $C_{A}$ and $C_{A^{\prime}}$ of the same color if $A \cap A^{\prime} \neq \emptyset$, and $C_{A}$ and $C_{A^{\prime}}$ can be combined into one c.a.h. cycle (with respect to colors in $A \cap A^{\prime}$ ) via Proposition 4.1 .

The construction for a c.a.h. cycle in $\mathfrak{D}^{*}$ proceeds as follows. Start with the set of cycles $\mathcal{C}=\left\{C_{A}: A \in \mathcal{A}\right\}$. As long as $\mathcal{C}$ contains more than one cycle, choose two cycles in $\mathcal{C}$, each containing an edge of the same color, and combine them. This can always be done unless the set $\mathcal{C}$ is reduced to a set of cycles, each containing edge colors that appear in no other cycles. However, this is impossible, since every pair of points (which corresponds to colors of edges) in $\mathfrak{D}^{*}$ appears in some block, and hence some cycle of $\mathcal{C}$. The result is therefore a c.a.h. cycle in $\mathfrak{D}^{*}$.

To seed the recursive constructions above, some small c.a.h. set systems are required. These are given in the next subsection.

\subsection{Small orders.}

Proposition 4.6. There exists a c.a.h. $\operatorname{STS}(n)$ for $n \in\{3,7,9,13,15\}$.

Proof. The proposition is trivially true for $n=3$. The required c.a.h. $\operatorname{STS}(n)$ for $n \in\{7,9,13,15\}$ are given in Appendix A.1.

Proposition 4.7. There exists an alternating hamiltonian minimum $(n, 3,2)$ covering for $n \in\{4,5,6,8,10,11,12,14,16,20\}$.

Proof. The required alternating hamiltonian minimum $(n, 3,2)$-coverings are given in Appendix A.2.

Proposition 4.8. There exists an alternating hamiltonian $\{3\}$-GDD of type $2^{3}$ and a c.a.h. $\{3\}$-GDD of the following types:
(i) $2^{4}$
(ii) $1^{6} 3^{1}$
(iii) $3^{3}$
(iv) $2^{3} 4^{1}$
(v) $2^{6}$
(vi) $1^{12} 3^{1} \quad$ (vii) $5^{3}$.

Proof. For the alternating hamiltonian $\{3\}$-GDD $(X, \mathcal{G}, \mathcal{A})$ of type $2^{3}$, take $X=[6]$, the groups to be $\{i, i+3\}, i \in[3]$, and the blocks to be $\{2,1,3\},\{3,4,5\},\{5,1,6\}$, and $\{6,4,2\}$.

The other required c.a.h. $\{3\}$-GDDs are given in Appendix A.3, noting the following.

For the c.a.h. $\{3\}$-GDD of type $2^{4}$, the groups are $\{i, i+4\}, i \in[4]$.

For the c.a.h. $\{3\}$-GDD of type $1^{6} 3^{1}$, the group of size three is $\{4,5,9\}$.

For the c.a.h. $\{3\}$-GDD of type $3^{3}$, the groups are $\{i, i+3, i+6\}, i \in[3]$.

For the c.a.h. $\{3\}$-GDD of type $2^{3} 4^{1}$, the groups are $\{1,5\},\{2,6\},\{3,7\},\{4,8$, $9,10\}$.

For the c.a.h. $\{3\}$-GDD of type $2^{6}$, the groups are $\{i, i+6\}, i \in[6]$.

For the c.a.h. $\{3\}$-GDD of type $1^{12} 3^{1}$, the group of size three is $\{3,8,14\}$.

For the c.a.h. $\{3\}$-GDD of type $5^{3}$, the groups are $\{i, i+3, i+6, i+9, i+12\}$, $i \in[3]$. 
Proposition 4.9. There exists a c.a.h. $\{3\}$-GDD of type $6^{t}$, for $t \in\{3,4,5,6$, $8\}$.

Proof. For $t \in\{3,4,6\}$, take a $\{3\}$-GDD of type $2^{t}$, which exists by Theorem 2.3 , as the master GDD and apply Theorem 4.5 with weight function $\omega(\cdot)=3$. The required ingredient, c.a.h. $\{3\}$-GDD of type $3^{3}$, exists by Proposition 4.8 ,

For $t \in\{5,8\}$, take a $\{4\}$-GDD of type $3^{t}$, which exists by Theorem 2.4, as the master GDD and apply Theorem 4.5 with weight function $\omega(\cdot)=2$. The required ingredient, c.a.h. $\{3\}$-GDD of type $2^{4}$, exists by Proposition 4.8

Proposition 4.10. There exists a c.a.h. $\{3\}$-GDD of type $6^{t} u^{1}$, for $n \in\{3,4,5$, $6,7,8,9,10,11,13,14,17,18,22\}$ and $u \in\{4,8\}$.

Proof. For $t \in\{5,6,10\}$ and $u \in\{4,8\}$, take the $\{4,7\}$-GDD of type $3^{t} u^{1}$ in Appendix B.2 as the master GDD, and apply Wilson's Fundamental Construction (Figure 11) with weight function $\omega$ that assigns weight zero to the $u / 2$ points $3 t+$ $u / 2+1,3 t+u / 2+2, \ldots, 3 t+u$ in the group of size $u$, and weight two to each of the remaining points. Use as ingredient GDDs an alternating hamiltonian $\{3\}$-GDD of type $2^{3}$ and c.a.h. $\{3\}$-GDDs of types $2^{4}$ and $2^{6}$, all of which exist by Proposition 4.8. The result is a $\{3\}$-GDD $\mathfrak{D}^{*}$ of type $6^{t} u^{1}$. Let $\mathcal{A}^{\prime}$ be the set of blocks, indicated in bold (and also in italics when $u=8$ ), of the master GDD. Then $\bigcup_{A \in \mathcal{A}^{\prime}} \mathcal{B}_{A}$ (with the notation in Figure 1), together with the blocks of $\mathfrak{D}^{*}$, is a c.a.h. cycle in $\mathfrak{D}^{*}$.

For $t \in\{3,4,7,8,11\}$ and $u=4$, take the $\{4\}$-GDD of type $3^{t+1}$ in Appendix B.1 as the master GDD and apply Wilson's Fundamental Construction (Figure 1) with weight function $\omega$ that assigns weight zero to the point $3(t+1)$ and weight two to each of the remaining points. Use as ingredient GDDs an alternating hamiltonian $\{3\}$-GDD of type $2^{3}$ and a c.a.h. $\{3\}$-GDD of type $2^{4}$, both of which exist by Proposition 4.8. The result is a $\{3\}$-GDD $\mathfrak{D}^{*}$ of type $6^{t} 4^{1}$. Let $\mathcal{A}^{\prime}$ be the set of blocks, indicated in bold, of the master GDD. Then $\bigcup_{A \in \mathcal{A}^{\prime}} \mathcal{B}_{A}$ (with the notation in Figure 1), together with the blocks of $\mathfrak{D}^{*}$, is a c.a.h. cycle in $\mathfrak{D}^{*}$.

For $t \in\{3,4,7,8,11\}$ and $u=8$, take a $\{4\}$-GDD of type $3^{t+1}$, which exists by Theorem 2.4, as the master GDD and apply Theorem 4.5 with a weight function that assigns weight four to one point and weight two to each of the remaining points. The required ingredient c.a.h. $\{3\}$-GDDs of types $2^{4}$ and $2^{3} 4^{1}$ exist by Proposition 4.8 .

For the remaining $t$ and $u$, we break into three cases.

$t \in\{9,18\}$ : Take a $\{3\}$-GDD of type $(t / 3)^{3}$, which exists by Theorem 2.4 as the master GDD and apply Theorem 4.5 with weight function $\omega(\cdot)=6$ to obtain a c.a.h. $\{3\}$-GDD $\mathfrak{S}$ of type $(2 t)^{3}$. The required ingredient c.a.h. $\{3\}$-GDD of type $6^{3}$ exists by Proposition [4.9. Now adjoin $u$ points and break up the groups of $\mathfrak{S}$ (Proposition 4.4) using a c.a.h. $\{3\}$-GDD of type $6^{t / 3} u^{1}$, whose existence has been established above, to obtain a c.a.h. $\{3\}$-GDD of type $6^{t} u^{1}$.

$t \in\{13,14\}:$ Take a $\{3\}$-GDD of type $6^{3}(2(t-9))^{1}$, which exists by Theorem 2.4. as the master GDD and apply Theorem 4.5. with weight function $\omega(\cdot)=3$ to obtain a c.a.h. $\{3\}$-GDD $\mathfrak{S}$ of type $18^{3}(6(t-9))^{1}$. The required ingredient c.a.h. $\{3\}$-GDD of type $3^{3}$ exists by Proposition 4.8, Now adjoin $u$ points and break up the groups of $\mathfrak{S}$ (Proposition 4.4) using c.a.h. $\{3\}$-GDDs of type $6^{s} u^{1}, s \in\{3, t-9\}$, whose existence has been established above, to obtain a c.a.h. $\{3\}$-GDD of type $6^{t} u^{1}$. 
$t \in\{17,22\}$ : Take a $\{3\}$-GDD of type $((t-2) / 5)^{4}((t+8) / 5)^{1}$, which exists by Theorem 2.4 as the master GDD and apply Theorem 4.5 with weight function $\omega(\cdot)=6$ to obtain a c.a.h. $\{3\}$-GDD $\mathfrak{S}$ of type $(6(t-2) / 5)^{4}(6(t+8) / 5)^{1}$. The required ingredient c.a.h. $\{3\}$-GDD of type $6^{3}$ exists by Proposition 4.8. Now adjoin $u$ points and break up the groups of $\mathfrak{S}$ (Proposition 4.4) using c.a.h. $\{3\}$-GDDs of type $6^{s} u^{1}, s \in\{(t-2) / 5,(t+8) / 5\}$, whose existence has been established above, to obtain a c.a.h. $\{3\}$-GDD of type $6^{t} u^{1}$.

\subsection{Piecing things together.}

Proposition 4.11. There exists a c.a.h. $\{3\}$-GDD of type $6^{t}$, for all $t \geq 3$.

Proof. Take a $\operatorname{PBD}(t,\{3,4,5,6,8\})$, which exists by Theorem 2.1, as the master GDD and apply Theorem 4.5 with weight function $\omega(\cdot)=6$. The required ingredient c.a.h. $\{3\}$-GDDs of type $6^{s}, s \in\{3,4,5,6,8\}$, exist by Proposition 4.9.

Proposition 4.12. There exists a c.a.h. $\{3\}$-GDD of type $6^{t} u^{1}$, for all $t \geq 3$ and $u \in\{4,8\}$.

Proof. For $t \in\{3,4,5,6,7,8,9,10,11,13,14,17,18,22\}$ and $u \in\{4,8\}$, the required GDDs exist by Proposition 4.10. For other values of $t$, take a $\operatorname{PBD}(t+1,\{4,5,6,7\})$, which exists by Theorem [2.2, as the master GDD and apply Theorem 4.5 with weight function $\omega$ that assigns weight six to each of $t$ points and weight $u$ to the remaining point. The required ingredient c.a.h. $\{3\}$-GDDs of types $6^{s}$ and $6^{s-1} u^{1}$, $s \in\{4,5,6,7\}$, exist by either Proposition 4.11 or Proposition 4.10

We are now ready to state the main result of this section.

Theorem 4.13. There exists an alternating hamiltonian minimum $(n, 3,2)$ covering, for all $n \geq 3$.

Proof. The existence of alternating hamiltonian minimum $(n, 3,2)$-coverings for $n \in$ $\{3,4, \ldots, 16\} \cup\{20\}$ has been established by either Proposition 4.6 or Proposition 4.7.

A c.a.h. minimum $(17,3,2)$-covering can be obtained by adjoining two points and filling in the groups of a c.a.h. $\{3\}$-GDD of type $5^{3}$ with a c.a.h. STS(7), which exist by Propositions 4.6 and 4.8 .

For $n \equiv 0(\bmod 6), n \geq 18$, take a c.a.h. $\{3\}$-GDD of type $6^{n / 6}$, which exists by Proposition 4.11, and fill in groups with a c.a.h. minimum $(6,3,2)$-covering, which exists by Proposition 4.7 .

For $n \equiv 1(\bmod 6), n \geq 19$, take a c.a.h. $\{3\}$-GDD of type $6^{(n-1) / 6}$, which exists by Proposition 4.11, adjoin one point and fill in groups with a c.a.h. STS(7), which exists by Proposition 4.6 .

For $n \equiv 2$ or $4(\bmod 6), n \geq 22$, take a c.a.h. $\{3\}$-GDD of type $6^{(n-8) / 6} 8^{1}$ or of type $6^{(n-4) / 6} 4^{1}$ (which exists by Proposition 4.12), respectively, and fill in groups with alternating hamiltonian minimum $(m, 3,2)$-coverings, $m \in\{4,6,8\}$, which exist by Proposition 4.7 .

For $n \equiv 3(\bmod 6), n \geq 21$, take a c.a.h. $\{3\}$-GDD of type $6^{(n-3) / 6}$, adjoin three points and fill in the groups with c.a.h. $\{3\}$-GDD of type $1^{6} 3^{1}$ and c.a.h. STS(9), which exist by Propositions 4.6 and 4.8 . 
For $n \equiv 5(\bmod 6), n \geq 23$, take a c.a.h. $\{3\}$-GDD of type $6^{(n-5) / 6} 4^{1}$, adjoin one point and fill in the groups with c.a.h. $\operatorname{STS}(7)$ and an alternating hamiltonian minimum $(5,3,2)$-covering, which exist by Propositions 4.6 and 4.7.

Corollary 4.14. There exists a minimum (n,3,2)-covering which possesses a 2shift universal cycle, for all $n \geq 3$.

\section{Application to 2-RAdius Sequences}

Jaromczyk and Lonc [24] studied sequences $\left(a_{1}, a_{2}, \ldots, a_{m}\right) \in[n]^{m}$ with the property that for every distinct $x, y \in[n]$, there exist $a_{i}$ and $a_{j}$ such that $\left\{a_{i}, a_{j}\right\}=$ $\{x, y\}$ and $|i-j| \leq k$. These sequences are known as $k$-radius sequences of order $n$. For given $n$ and $k$, the objective is to find a shortest (optimal) $k$-radius sequence of order $n$. The length of such a sequence is denoted $f_{k}(n)$.

Motivation for studying short $k$-radius sequences comes from computation where a certain two-argument function must be evaluated for all pairs of $n$ large objects $\mathcal{O}_{1}, \mathcal{O}_{2}, \ldots, \mathcal{O}_{n}$, which are too large to be all held in fast primary memory (such as internal memory in the I/O model of Aggarwal and Vitter 11, and cache memory in the cache model of Sen et al. 28) at once. Hence, instead of the simple twoloop algorithm that iterates through all the pairs of objects, a schedule to determine which objects are to be fetched into memory and which are to be replaced is needed. This schedule must ensure that for all pairs of objects $x$ and $y$, there is some point in time where $x$ and $y$ are both in memory. If the memory can only hold $k+1$ objects at any one time, such a schedule corresponds to a $k$-radius sequence $\left(a_{1}, a_{2}, \ldots, a_{m}\right)$ of order $n$, assuming a first-in-first-out (FIFO) object replacement strategy 24]: initially (at time step one) the objects $\mathcal{O}_{a_{1}}, \mathcal{O}_{a_{2}}, \ldots, \mathcal{O}_{a_{k+1}}$ reside in primary memory, and at step time step $t \geq 2$ the object $\mathcal{O}_{a_{k+t}}$ is fetched to replace object $\mathcal{O}_{a_{t-1}}$. For reasons of efficiency, short schedules are desirable.

Extending beyond cache algorithms, $k$-radius sequences are also applicable when the large objects reside in remote servers and local storage is not large enough to store all the objects for computations required to run over all possible pairs of objects. For bandwidth efficiency, we would like to minimize the fetching of the large objects over the network into local storage. An example of this scenario is the restoration of the order of images in a sequence of MRI slices, when the MRI images (which are typically quite large) reside in remote databases [14.

The function $f_{1}(n)$ has been completely determined by Ghosh [13] in the context of database theory.

Theorem 5.1 (Ghosh [13]).

$$
f_{1}(n)= \begin{cases}\left(\begin{array}{l}
n \\
2
\end{array}\right)+1, & \text { if } n \text { is odd } \\
\left(\begin{array}{l}
n \\
2
\end{array}\right)+\frac{1}{2} n, & \text { if } n \text { is even. }\end{cases}
$$

The function $f_{2}(n)$ has been recently investigated by Jaromczyk and Lonc [24], who established the bounds below.

Theorem 5.2 (Jaromczyk and Lonc [24]).

$$
L(n) \leq f_{2}(n) \leq \frac{1}{2}\left(\begin{array}{l}
n \\
2
\end{array}\right)+\frac{10 n^{2}}{\log _{2} n}+2 n^{1.64},
$$


TABLE 1. New optimal 2-radius sequences of order $n$

\begin{tabular}{|c|c|c|}
\hline$n$ & length & sequence \\
\hline 8 & 17 & $(3,6,2,7,8,5,6,4,1,8,7,3,4,2,5,1,3)$ \\
\hline 10 & 30 & $(2,6,8,10,9,1,5,4,7,10,2,6,4,5,9,2,1,3,10,5,8,7,9,3,6,7,1,8,4,3)$ \\
\hline 11 & 33 & $(10,9,7,11,2,3,1,10,11,8,6,3,1,7,4,11,3,5,9,6,4,10,2,5,6,4,7,8,5,9,1,2,8)$ \\
\hline 12 & 37 & $\begin{array}{l}(12,6,7,11,10,9,3,6,5,2,9,1,8,5,7,3,12,1,10,6,4,8,3,11,2,1,7,4,9,12,8,2, \\
10,4,5,11,12)\end{array}$ \\
\hline 14 & 56 & $\begin{array}{l}(13,9,8,12,2,5,3,11,13,14,4,9,10,1,6,14,8,3,7,10,11,8,14,5,7,13,12,6,4,8, \\
1,3,6,2,11,9,12,3,10,4,13,2,1,7,11,4,1,5,12,10,14,2,5,9,6,7)\end{array}$ \\
\hline 15 & 60 & $\begin{array}{l}(10,9,7,1,11,14,9,15,12,2,8,6,13,7,5,3,15,6,11,12,5,14,2,3,10,12,1,13,3 \\
4,11,15,8,10,5,6,9,3,8,5,1,4,2,6,14,1,13,15,7,8,14,4,10,11,13,2,9,7,4 \\
12)\end{array}$ \\
\hline 16 & 65 & $\begin{array}{l}(7,8,9,6,10,4,15,13,1,6,12,3,15,8,16,12,10,5,2,15,6,11,16,2,13,9,12,16 \\
14,4,3,5,11,8,10,13,7,3,15,9,14,11,13,7,5,16,1,3,10,2,14,8,4,1,2,7,12,4 \\
11,9,1,5,14,6,7)\end{array}$ \\
\hline 18 & 90 & $\begin{array}{l}(2,9,7,1,17,5,12,13,4,17,9,18,6,1,15,8,13,16,6,4,7,1,16,10,18,17,6,14,11, \\
7,12,10,14,15,13,11,16,3,15,12,18,4,3,10,6,1,12,14,8,5,18,7,13,8,9,10,3, \\
13,1,2,11,8,17,3,5,2,6,8,11,4,14,2,18,10,11,5,9,16,15,2,17,12,16,9,14,3, \\
7,15,4,5)\end{array}$ \\
\hline
\end{tabular}

TABle 2. New short 2-radius sequences of order $n$

\begin{tabular}{|c|c|c|}
\hline$n$ & length & sequence \\
\hline 9 & 21 & $(9,3,2,7,8,4,9,3,1,8,5,9,7,6,1,2,4,5,6,3,8)$ \\
\hline 13 & 42 & $\begin{array}{l}(4,9,11,5,7,4,8,2,11,3,1,4,12,10,11,13,6,4,9,1,8,10,6,3,12,7,9,10,2,5,6, \\
1,7,2,13,12,5,8,3,13,9,1)\end{array}$ \\
\hline 17 & 73 & $\begin{array}{l}(1,2,3,4,5,1,6,7,3,8,9,1,10,11,3,12,13,1,14,15,3,16,17,2,5,7,9,4,6,8,2,11, \\
13,4,10,12,5,8,13,15,6,10,14,2,9,15,12,7,14,11,5,15,17,4,14,16,8,10,17,7, \\
13,16,9,11,17,6,12,16,2,5,1,17,16)\end{array}$ \\
\hline
\end{tabular}

where

$$
L(n)=\left\{\begin{array}{lll}
\frac{1}{2}\left(\begin{array}{l}
n \\
2
\end{array}\right)+\frac{1}{4} n+1, & \text { if } n \equiv 0 \quad(\bmod 4), \\
\frac{1}{2}\left(\begin{array}{l}
n \\
2
\end{array}\right)+2, & \text { if } n \equiv 1 \quad(\bmod 4), \\
\frac{1}{2}\left(\begin{array}{l}
n \\
2
\end{array}\right)+\frac{3}{4} n, & \text { if } n \equiv 2 \quad(\bmod 4), \\
\frac{1}{2}\left(\begin{array}{l}
n \\
2
\end{array}\right)+\frac{1}{2} n, & \text { if } n \equiv 3 \quad(\bmod 4) .
\end{array}\right.
$$

The upper bound in Theorem 5.2 comes from a number-theoretic construction 24, Section 2], which we refer to as the Jaromczyk-Lonc construction. Exact values of $f_{2}(n)$ have been previously known only for $n \leq 7$ [24, 15]. Next, $f_{2}(n)$ is determined for $n \in\{8,10,11,14,15\}$ and better upper bounds on $f_{2}(n)$, for $n \in\{9,12,13,16,17,18\}$, are given.

5.1. Some new 2-radius sequences. The value of $f_{2}(n)$ meets the lower bound in Theorem 5.2 for $n \in\{8,10,11,14,15\}$. The new optimal 2-radius sequences proving this are given in Table 1 .

When $n \in\{9,12,13,16,17,18\}$, we improve on the shortest 2-radius sequence of order $n$ currently known 24. The new 2-radius sequences are given in Table 2. These sequences are not known to be optimal.

The new 2-radius sequences obtained above are all found through a computer search. The state of knowledge for small values of $n$ is provided in Table 3 , where an entry with a single number gives the exact value of $f_{2}(n)$ and an entry of the form " $a-b$ " means that the corresponding value of $f_{2}(n)$ lies between $a$ and $b$ (inclusive). 
TABLE 3. State of knowledge of $f_{2}(n), 2 \leq n \leq 18$

\begin{tabular}{|c|ccccccccccccccccc|}
\hline$n$ & 2 & 3 & 4 & 5 & 6 & 7 & 8 & 9 & 10 & 11 & 12 & 13 & 14 & 15 & 16 & 17 & 18 \\
\hline$f_{2}(n)$ & 2 & 3 & 5 & 7 & 12 & 14 & $\mathbf{1 7}$ & $\mathbf{2 0}-\mathbf{2 1}$ & $\mathbf{3 0}$ & $\mathbf{3 3}$ & $\mathbf{3 7}$ & $\mathbf{4 1 - 4 2}$ & $\mathbf{5 6}$ & $\mathbf{6 0}$ & $\mathbf{6 5}$ & $\mathbf{7 0}-\mathbf{7 3}$ & $\mathbf{9 0}$ \\
\hline
\end{tabular}

A bold entry indicates new results obtained in this paper, while the other entries are from [24, 15.

We now give details of our search procedure. The framework used is hillclimbing. To construct a 2-radius sequence of order $n$ and length $m$, we start with a random sequence in $[n]^{m}$ and modify it iteratively to get "closer" and "closer" to a 2-radius sequence, until either we end up with a 2-radius sequence or we get stuck. We measure the "closeness" of a sequence $S=\left(a_{1}, a_{2}, \ldots, a_{m}\right) \in[n]^{m}$ to a 2-radius sequence by its defect, denoted $\operatorname{def}(S)$, defined as the number of pairs $\{x, y\} \subseteq[n]$ for which there do not exist $a_{i}, a_{j}$ such that $\left\{a_{i}, a_{j}\right\}=\{x, y\}$ and $|i-j| \leq 2$. A sequence is therefore a 2-radius sequence if and only if it has zero defect. At each step, modification of a sequence $\left(a_{1}, a_{2}, \ldots, a_{m}\right)$ proceeds as follows. We select three distinct positions $i, j, k \in[m]$ and replace the values $a_{i}, a_{j}$, and $a_{k}$ by elements of $[n]$, drawn uniformly at random. If this modification does not result in a new sequence of higher defect, we accept the modification. Otherwise, we reject the modification. The procedure is terminated when we have a sequence of zero defect, and it is restarted after a prespecified period of time without finding a defectreducing modification. This hillclimbing procedure is described more formally in pseudocode in Algorithm 1,

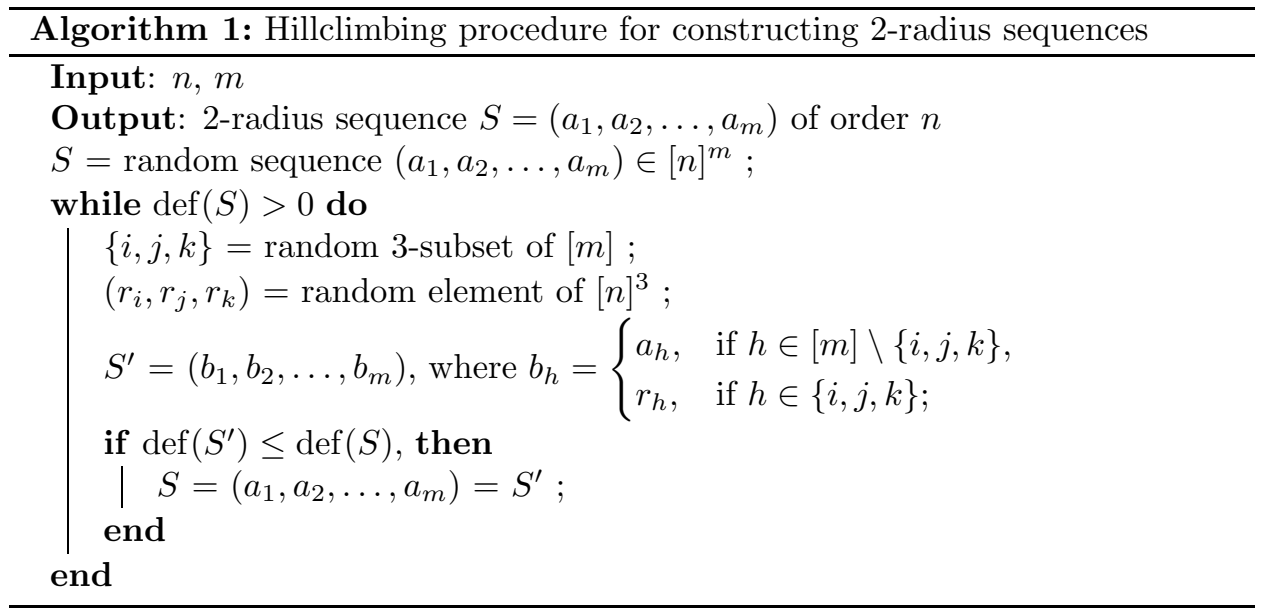

All the 2-radius sequences in Tables 1 and 2 are obtained using Algorithm 1.

5.2. Improvements to theoretically provable bounds. Although the bounds in Theorem 5.2 are asymptotically tight, proving $f_{2}(n)=(1+o(1)) n^{2} / 4$, the upper bound is rather weak. Gilkerson et al. [15] proved the following upper bound, which, although not asymptotically tight, improves on the upper bound in Theorem 5.2 for $n \leq 1.329 \times 10^{36}$.

Theorem 5.3 (Gilkerson et al. 15]). Let $m^{2} \mid n$ be such that there exists either a $\operatorname{PBD}\left(m^{2},\{m\}\right)$ (affine plane of order $m$ ) or a $\operatorname{PBD}\left(m^{2}-m+1,\{m\}\right)$ (projective plane of order $m-1)$. Then $f_{2}(n) \leq n^{2} / 3+n$. 
However, at present, $\operatorname{PBD}\left(m^{2},\{m\}\right)$ and $\operatorname{PBD}\left(m^{2}-m+1,\{m\}\right)$ are known to exist only when $m$ or $m-1$ is a prime power, respectively (see, for example, [29]). Hence, the applicability of Theorem 5.3 is limited. The results in $\$ 4$ imply the following stronger result.

Theorem 5.4. For all $n \geq 3, f_{2}(n) \leq 2 C(n, 3,2)+1$.

Proof. Follows directly from Corollary 4.14 and the observation that if $\left(u_{0}, u_{1}, \ldots\right.$, $\left.u_{2 m-1}\right)$ is a 2 -shift universal cycle for an $(n, 3,2)$-covering, then $\left(u_{0}, u_{1}, \ldots, u_{2 m-1}\right.$, $\left.u_{0}\right)$ is a 2 -radius sequence of order $n$ and length $2 m+1$.

The upper bound in Theorem 5.4 is strictly better than the upper bound in Theorem 5.3 for all $n \geq 3$, since

$$
\left(\frac{n^{2}}{3}+n\right)-(2 C(n, 3,2)+1)= \begin{cases}\frac{4 n}{3}-1, & \text { if } n \equiv 1 \text { or } 3 \quad(\bmod 6) \\ n-\frac{5}{3}, & \text { if } n \equiv 2 \text { or } 4 \quad(\bmod 6) \\ \frac{4 n-7}{3}, & \text { if } n \equiv 5 \quad(\bmod 6) \\ n-1, & \text { if } n \equiv 0 \quad(\bmod 6)\end{cases}
$$

It is also better than the upper bound in Theorem 5.2 for all $n \leq 1.329 \times 10^{36}$.

5.3. Actual performance of constructions. The upper bound in Theorem 5.2 is what is theoretically provable in the Jaromczyk-Lonc construction of 2-radius sequences. However, its actual performance is much better. We provide in Table 4 a comparison of the lengths of 2-radius sequences actually produced by the Jaromczyk-Lonc construction and that obtained through our bound in Theorem 5.4. In Table 4.

TABle 4. Performance comparison for constructions of 2-radius sequences of order $n, 9 \leq n \leq 44$

\begin{tabular}{|l|cccccccccccc|}
\hline$n$ & 9 & 10 & 11 & 12 & 13 & 14 & 15 & 16 & 17 & 18 & 19 & 20 \\
\hline len JL theory & 346 & 410 & 479 & 552 & 629 & 711 & 798 & 888 & 983 & 1082 & 1185 & 1292 \\
\hline len $_{\text {JL actual }}$ & 37 & 49 & 39 & 53 & 45 & 62 & 80 & 99 & 76 & 98 & 105 & 129 \\
\hline len $_{\text {this }}$ & $\mathbf{2 5}$ & $\mathbf{3 5}$ & 39 & $\mathbf{4 9}$ & 53 & 67 & $\mathbf{7 1}$ & $\mathbf{8 7}$ & 93 & 109 & 115 & 135 \\
\hline
\end{tabular}

\begin{tabular}{|l|cccccccccccc|}
\hline$n$ & 21 & 22 & 23 & 24 & 25 & 26 & 27 & 28 & 29 & 30 & 31 & 32 \\
\hline len JL theory $^{1403}$ & 1518 & 1638 & 1761 & 1888 & 2019 & 2153 & 2292 & 2434 & 2580 & 2730 & 2884 \\
\hline len JL actual $^{1}$ & 158 & 185 & 150 & 179 & 170 & 202 & 256 & 290 & 217 & 254 & 297 & 336 \\
\hline len $_{\text {this }}$ & $\mathbf{1 4 1}$ & $\mathbf{1 6 3}$ & 171 & 193 & 201 & 227 & $\mathbf{2 3 5}$ & $\mathbf{2 6 3}$ & 273 & 301 & 311 & 343 \\
\hline
\end{tabular}

\begin{tabular}{|l|cccccccccccc|}
\hline$n$ & 33 & 34 & 35 & 36 & 37 & 38 & 39 & 40 & 41 & 42 & 43 & 44 \\
\hline len JL theory $^{3041}$ & 3202 & 3366 & 3535 & 3707 & 3882 & 4061 & 4244 & 4430 & 4620 & 4813 & 5010 \\
\hline len $_{\text {JL actual }}$ & 382 & 424 & 361 & 405 & 351 & 398 & 446 & 495 & 430 & 482 & 540 & 594 \\
\hline len $_{\text {this }}$ & $\mathbf{3 5 3}$ & $\mathbf{3 8 7}$ & 399 & 433 & 445 & 483 & 495 & 535 & 549 & 589 & 603 & 647 \\
\hline
\end{tabular}


$\operatorname{len}_{\mathrm{JL}}$ theory $=$ theoretically provable length of 2-radius sequence of order $n$ produced by the Jaromczyk-Lonc construction,

len $_{\mathrm{JL} \text { actual }}=$ actual length of 2-radius sequence of order $n$ produced by the Jaromczyk-Lonc construction,

$\operatorname{len}_{\text {this }}=$ actual (and also theoretically provable) length of 2-radius sequence of order $n$ produced by our construction in this paper.

Entries in bold denote orders for which our construction outperforms the actual performance of the Jaromczyk-Lonc construction.

5.4. Recent advancements. Blackburn and Mckee [4] have very recently revealed unexpected connections between $k$-radius sequences, lattice tilings, and logarithms in $\mathbb{Z}_{k}$. In particular, they obtained an upper bound on $f_{2}(n)$ that improves on Theorem 5.2 .

\section{Conclusion}

A new ordering on the blocks of set systems, called shift universal cycles, is introduced. Minimum $(n, 3,2)$-coverings that admit 2-shift universal cycles are shown to exist for all $n$. These minimum $(n, 3,2)$-coverings are used to construct short 2-radius sequences, which have applications in cache algorithms.

\section{Appendix A. Some C.A.H. Set Systems}

A hamiltonian cycle of length $m$ in the block intersection graph of a set system or GDD, $\mathfrak{S}$, is specified as a sequence of blocks in $\mathfrak{S}: A_{0} A_{1} \cdots A_{m-1}$. The edges of the hamiltonian cycle is taken to be $\left\{A_{i}, A_{i+1}\right\}, i \in \mathbb{Z}_{m}$.

In each case, the set of points is taken to be $[n]$.

\section{A.1. Some small c.a.h. $\operatorname{STS}(n)$.}

\begin{tabular}{|l|l|}
\hline$n$ & c.a.h. cycle in STS $(n)$ \\
\hline 7 & $\{5,3,1\}\{1,7,4\}\{4,2,3\}\{3,6,7\}\{7,5,2\}\{2,1,6\}\{6,4,5\}$ \\
\hline 9 & $\{3,6,9\}\{9,5,1\}\{1,6,8\}\{8,7,9\}\{9,4,2\}\{2,8,5\}\{5,3,7\}\{7,4,1\}\{1,3,2\}\{2,7,6\}$ \\
& $\{6,5,4\}\{4,8,3\}$ \\
\hline 13 & $\{5,10,3\}\{3,13,12\}\{12,7,5\}\{5,8,4\}\{4,11,6\}\{6,2,3\}\{3,8,1\}\{1,7,9\}\{9,6,5\}$ \\
& $\{5,1,2\}\{2,10,8\}\{8,12,9\}\{9,2,4\}\{4,12,10\}\{10,6,7\}\{7,8,11\}\{11,3,9\}\{9,13,10\}$ \\
& $\{10,1,11\}\{11,12,2\}\{2,13,7\}\{7,3,4\}\{4,13,1\}\{1,12,6\}\{6,8,13\}\{13,11,5\}$ \\
\hline 15 & $\{7,11,1\}\{1,10,5\}\{5,6,15\}\{15,8,11\}\{11,2,4\}\{4,13,8\}\{8,14,1\}\{1,9,12\}\{12,6,11\}$ \\
& $\{11,5,13\}\{13,14,12\}\{12,4,15\}\{15,1,2\}\{2,3,12\}\{12,7,10\}\{10,11,9\}\{9,13,6\}$ \\
& $\{6,7,8\}\{8,10,2\}\{2,14,6\}\{6,4,1\}\{1,13,3\}\{3,11,14\}\{14,15,9\}\{9,7,4\}\{4,10,14\}$ \\
& $\{14,5,7\}\{7,3,15\}\{15,13,10\}\{10,6,3\}\{3,4,5\}\{5,12,8\}\{8,3,9\}\{9,5,2\}\{2,13,7\}$ \\
\hline
\end{tabular}


A.2. Some small alternating Hamiltonian minimum $(n, 3,2)$-coverings.

The alternating hamiltonian minimum $(n, 3,2)$-coverings given below for $n \in$ $\{6,8,10,11,12,14,16,20\}$ are in fact c.a.h.

\begin{tabular}{|l|l|}
\hline$n$ & alternating hamiltonian cycle in minimum $(n, 3,2)$-coverings \\
\hline 4 & $\{3,1,2\}\{2,4,1\}\{1,4,3\}$ \\
\hline 5 & $\{1,2,3\}\{3,4,5\}\{5,2,4\}\{4,5,1\}$ \\
\hline 6 & $\{6,3,1\}\{1,4,2\}\{2,5,3\}\{3,6,4\}\{4,1,5\}\{5,2,6\}$ \\
\hline 8 & $\{3,8,1\}\{1,4,2\}\{2,5,3\}\{3,6,4\}\{4,8,5\}\{5,1,6\}\{6,2,8\}\{8,1,7\}\{7,4,5\}\{5,6,7\}$ \\
& $\{7,2,3\}$ \\
\hline 10 & $\{7,9,8\}\{8,4,3\}\{3,6,9\}\{9,10,8\}\{8,6,1\}\{1,10,2\}\{2,8,5\}\{5,1,9\}\{9,2,4\}\{4,1,7\}$ \\
& $\{7,8,10\}\{10,4,3\}\{3,1,2\}\{2,7,6\}\{6,10,5\}\{5,3,7\}$ \\
\hline 11 & $\{8,11,3\}\{3,6,10\}\{10,4,9\}\{9,8,1\}\{1,3,2\}\{2,7,10\}\{10,1,11\}\{11,7,5\}\{5,9,6\}$ \\
& $\{6,11,4\}\{4,1,2\}\{2,6,8\}\{8,10,5\}\{5,2,1\}\{1,6,7\}\{7,9,3\}\{3,5,4\}\{4,7,8\}$ \\
\hline 12 & $\{11,6,4\}\{4,5,12\}\{12,3,1\}\{1,8,9\}\{9,11,2\}\{2,12,3\}\{3,11,8\}\{8,12,9\}\{9,4,10\}$ \\
& $\{10,7,2\}\{2,1,5\}\{5,9,6\}\{6,12,7\}\{7,5,11\}\{11,1,10\}\{10,5,8\}\{8,6,2\}\{2,4,1\}$ \\
& $\{1,6,7\}\{7,9,3\}\{3,6,10\}\{10,12,11\}$ \\
\hline 14 & $\{9,10,13\}\{13,12,14\}\{14,9,10\}\{10,3,5\}\{5,8,4\}\{4,13,1\}\{1,8,3\}\{3,6,2\}\{2,7,13\}$ \\
$\{13,5,11\}\{11,6,4\}\{4,3,14\}\{14,1,2\}\{2,10,8\}\{8,12,9\}\{9,6,5\}\{5,2,1\}\{1,10,11\}$ \\
$\{11,14,12\}\{12,4,10\}\{10,6,7\}\{7,8,11\}\{11,2,12\}\{12,3,13\}\{13,6,8\}\{8,7,14\}$ \\
$\{14,5,6\}\{6,12,1\}\{1,7,9\}\{9,2,4\}\{4,7,3\}\{3,11,9\}$ \\
\hline 16 & $\{7,6,8\}\{8,3,9\}\{9,7,4\}\{4,13,8\}\{8,14,1\}\{1,9,12\}\{12,11,6\}\{6,14,2\}\{2,13,7\}$ \\
$\{7,10,12\}\{12,14,13\}\{13,11,5\}\{5,10,1\}\{1,4,6\}\{6,5,16\}\{16,1,2\}\{2,8,10\}$ \\
$\{10,9,11\}\{11,7,1\}\{1,15,2\}\{2,3,12\}\{12,4,15\}\{15,5,6\}\{6,13,9\}\{9,14,15\}$ \\
$\{15,11,8\}\{8,7,16\}\{16,9,10\}\{10,4,14\}\{14,16,13\}\{13,1,3\}\{3,14,11\}\{11,16,12\}$ \\
$\{12,8,5\}\{5,7,14\}\{14,15,16\}\{16,3,4\}\{4,11,2\}\{2,9,5\}\{5,4,3\}\{3,6,10\}$ \\
$\{10,13,15\}\{15,3,7\}$ \\
20 & $\{12,5,3\}\{3,14,8\}\{8,13,19\}\{19,11,5\}\{5,8,4\}\{4,2,11\}\{11,12,15\}\{15,14,18\}$ \\
$\{18,1,8\}\{8,10,17\}\{17,3,9\}\{9,19,2\}\{2,3,6\}\{6,8,15\}\{15,10,2\}\{2,7,13\}$ \\
$\{13,14,20\}\{20,1,2\}\{2,18,17\}\{18,13,14\}\{14,1,9\}\{9,13,10\}\{10,5,16\}\{16,9,7\}$ \\
$\{7,19,17\}\{17,5,15\}\{15,7,1\}\{1,3,10\}\{10,6,7\}\{7,11,8\}\{8,2,16\}\{16,13,12\}$ \\
$\{12,4,17\}\{17,18,20\}\{20,9,10\}\{10,4,18\}\{18,13,5\}\{5,6,9\}\{9,4,15\}\{15,20,16\}$ \\
$\{16,3,11\}\{11,10,14\}\{14,16,4\}\{4,7,3\}\{3,19,18\}\{18,6,16\}\{16,1,17\}\{17,11,6\}$ \\
$\{6,13,4\}\{4,3,20\}\{20,19,18\}\{18,7,12\}\{12,2,14\}\{14,7,5\}\{5,2,1\}\{1,6,12\}$ \\
$\{12,8,9\}\{9,18,11\}\{11,12,20\}\{20,5,6\}\{6,14,19\}\{19,16,15\}\{15,3,13\}\{13,11,1\}$ \\
$\{1,4,19\}\{19,10,12\}$ \\
\end{tabular}

\section{A.3. Some small c.a.h. $\{3\}$-GDDs.}

\begin{tabular}{|c|l|}
\hline$T$ & c.a.h. cycle in $\{3\}$-GDD of type $T$ \\
\hline $2^{4}$ & $\{2,3,1\}\{1,8,7\}\{7,2,4\}\{4,1,6\}\{6,7,5\}\{5,4,3\}\{3,6,8\}\{8,5,2\}$ \\
\hline $1^{6} 3^{1}$ & $\{8,6,4\}\{4,1,7\}\{7,9,2\}\{2,4,3\}\{3,6,9\}\{9,8,1\}\{1,3,5\}\{5,8,2\}\{2,1,6\}\{6,5,7\}$ \\
& $\{7,3,8\}$ \\
\hline $3^{3}$ & $\{5,6,7\}\{7,3,8\}\{8,1,9\}\{9,7,2\}\{2,4,3\}\{3,5,1\}\{1,2,6\}\{6,8,4\}\{4,9,5\}$ \\
\hline $2^{3} 4^{1}$ & $\{4,2,1\}\{1,8,3\}\{3,2,9\}\{9,6,1\}\{1,7,10\}\{10,5,2\}\{2,7,8\}\{8,5,6\}\{6,10,3\}$ \\
& $\{3,4,5\}\{5,9,7\}\{7,6,4\}$ \\
\hline $2^{6}$ & $\{3,2,1\}\{1,5,4\}\{4,2,6\}\{6,8,1\}\{1,10,9\}\{9,2,11\}\{11,1,12\}\{12,10,2\}\{2,5,7\}$ \\
& $\{7,4,3\}\{3,5,12\}\{12,9,4\}\{4,8,11\}\{11,3,6\}\{6,7,9\}\{9,8,5\}\{5,6,10\}\{10,11,7\}$ \\
& $\{7,12,8\}\{8,10,3\}$ \\
\hline $1^{12} 3^{1}$ & $\{11,15,5\}\{5,13,1\}\{1,9,12\}\{12,3,5\}\{5,14,9\}\{9,15,2\}\{2,10,13\}\{13,12,7\}$ \\
& $\{7,6,1\}\{1,3,2\}\{2,8,12\}\{12,6,14\}\{14,15,13\}\{13,4,3\}\{3,15,7\}\{7,8,9\}$ \\
& $\{9,4,10\}\{10,8,5\}\{5,7,2\}\{2,14,4\}\{4,12,15\}\{15,1,10\}\{10,12,11\}\{11,3,9\}$ \\
& $\{9,6,13\}\{13,11,8\}\{8,15,6\}\{6,5,4\}\{4,8,1\}\{1,14,11\}\{11,4,7\}\{7,14,10\}$ \\
& $\{10,3,6\}\{6,2,11\}$ \\
\hline $5^{3}$ & $\{2,3,1\}\{1,11,6\}\{6,4,5\}\{5,9,1\}\{1,8,15\}\{15,2,4\}\{4,9,14\}\{14,1,12\}\{12,8,4\}$ \\
& $\{4,11,3\}\{3,5,7\}\{7,8,9\}\{9,10,2\}\{2,12,7\}\{7,14,6\}\{6,10,8\}\{8,13,3\}$ \\
& $\{3,10,14\}\{14,15,13\}\{13,5,12\}\{12,11,10\}\{10,5,15\}\{15,7,11\}\{11,9,13\}$ \\
& $\{13,6,2\}$ \\
\hline
\end{tabular}




\section{Appendix B. Some GDDs}

\section{B.1. Some small $\{4\}$-GDDs of type $3^{t}$.}

Each of the $\{4\}$-GDDs of type $3^{t}$ listed in this section is on the set of points [3t], with groups $\{i, i+t, i+2 t\}, i \in[t]$.

\begin{tabular}{|c|c|}
\hline$T$ & $\{4\}$-GDD of type $T$ \\
\hline $3^{4}$ & $\begin{array}{l}\{\mathbf{1}, \mathbf{2}, \mathbf{3}, \mathbf{4}\}\{\mathbf{1}, \mathbf{6}, \mathbf{7}, \mathbf{8}\}\{\mathbf{2}, \mathbf{8}, \mathbf{9}, \mathbf{1 1}\}\{\mathbf{3}, \mathbf{5}, \mathbf{8}, \mathbf{1 0}\}\{\mathbf{4}, \mathbf{5}, \mathbf{6}, \mathbf{1 1}\}\{\mathbf{4}, \mathbf{7}, \mathbf{9}, \mathbf{1 0}\}\{\mathbf{2}, \mathbf{5}, \mathbf{7}, \mathbf{1 2}\} \\
\{1,10,11,12\}\{3,6,9,12\}\end{array}$ \\
\hline $3^{5}$ & $\begin{array}{l}\{\mathbf{1}, \mathbf{2}, \mathbf{4}, \mathbf{8}\}\{\mathbf{2}, \mathbf{3}, \mathbf{5}, \mathbf{9}\}\{\mathbf{3}, \mathbf{4}, \mathbf{6}, \mathbf{1 0}\}\{\mathbf{4}, \mathbf{5}, \mathbf{7}, \mathbf{1 1}\}\{\mathbf{5 , 6 , 8} \mathbf{1 2}\}\{\mathbf{6}, \mathbf{7}, \mathbf{9}, \mathbf{1 3}\} \\
\{\mathbf{7}, \mathbf{8}, \mathbf{1 0}, \mathbf{1 4}\}\{2,10,11,13\}\{1,9,10,12\}\{3,11,12,14\}\{4,12,13,15\}\{1,5,13,14\} \\
\{2,6,14,15\}\{1,3,7,15\}\{8,9,11,15\}\end{array}$ \\
\hline $3^{8}$ & $\begin{array}{l}\{\mathbf{9}, \mathbf{1 4}, \mathbf{1 5}, \mathbf{1 9}\}\{\mathbf{1}, \mathbf{2}, \mathbf{5}, \mathbf{1 9}\}\{\mathbf{1}, \mathbf{3}, \mathbf{8}, \mathbf{1 2}\}\{\mathbf{2}, \mathbf{3}, \mathbf{6}, \mathbf{2 0}\}\{\mathbf{2}, \mathbf{4}, \mathbf{1 3}, \mathbf{1 6}\}\{\mathbf{3}, \mathbf{4}, \mathbf{7}, \mathbf{2 1}\} \\
\{\mathbf{4}, \mathbf{5}, \mathbf{9}, \mathbf{2 2}\}\{\mathbf{5}, \mathbf{6}, \mathbf{1 0}, \mathbf{2 3}\}\{\mathbf{1}, \mathbf{6}, \mathbf{7}, \mathbf{1 1}\}\{\mathbf{1 1}, \mathbf{1 7}, \mathbf{1 8}, \mathbf{2 1}\}\{2,7,9,12\}\{3,9,10,13\} \\
\{4,10,11,14\}\{5,11,12,15\}\{6,12,13,17\}\{7,13,14,18\}\{10,15,17,20\}\{12,18,19,22\} \\
\{13,19,20,23\}\{1,14,20,21\}\{2,15,21,22\}\{3,17,22,23\}\{1,4,18,23\}\{5,8,18,20\} \\
\{4,17,19,24\}\{3,15,16,18\}\{2,8,14,17\}\{1,13,15,24\}\{12,14,16,23\}\{2,11,23,24\} \\
\{1,10,16,22\}\{8,9,21,23\}\{7,20,22,24\}\{6,16,19,21\}\{8,11,13,22\}\{10,12,21,24\} \\
\{9,11,16,20\}\{7,8,10,19\}\{6,9,18,24\}\{5,7,16,17\}\{4,6,8,15\}\{3,5,14,24\}\end{array}$ \\
\hline $3^{9}$ & 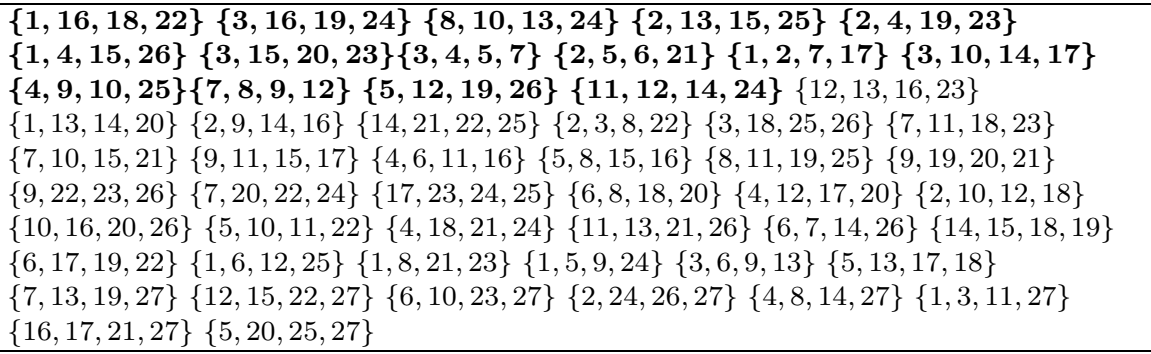 \\
\hline $3^{12}$ & 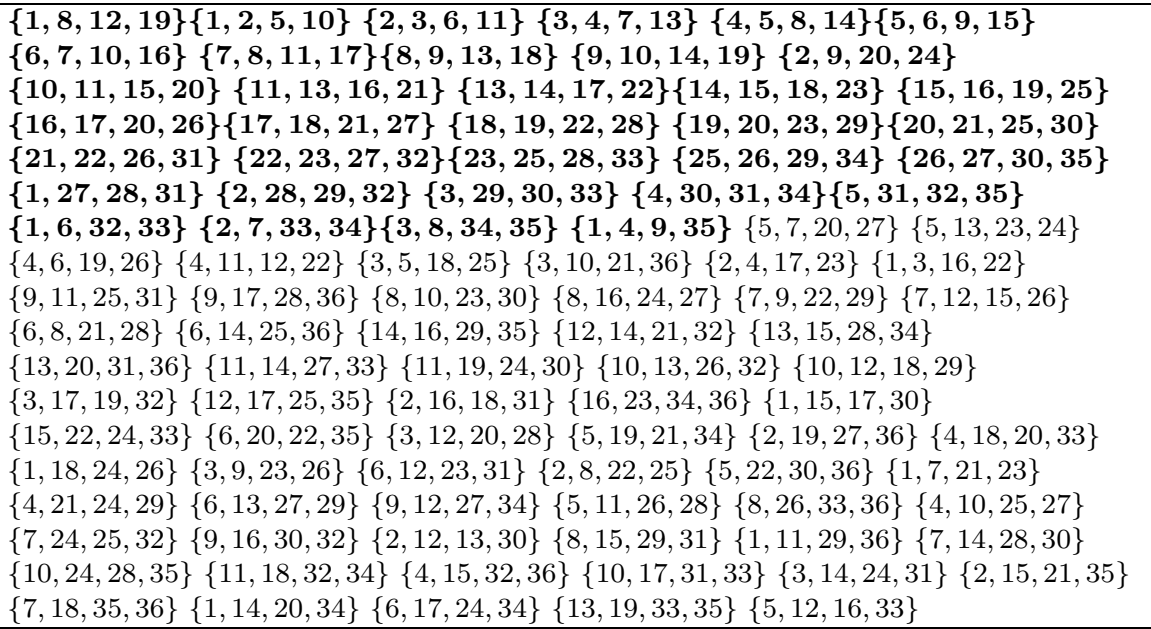 \\
\hline
\end{tabular}


B.2. Some small $\{4,7\}$-GDDs of type $3^{t} u^{1}$. Each of the $\{4,7\}$-GDDs of type $3^{t} u^{1}$ listed in this section is on the set of points $[3 t+u]$, with groups $\{3 i-2,3 i-1,3 i\}$, $i \in[t]$, and $\{3 t+1,3 t+2, \ldots, 3 t+u\}$.

\begin{tabular}{|c|c|}
\hline$T$ & $\{4,7\}$-GDD of type $T$ \\
\hline $3^{5} 6^{1}$ & $\begin{array}{l}\{\mathbf{1}, \mathbf{4}, \mathbf{1 4}, \mathbf{1 6}\}\{\mathbf{2}, \mathbf{1 1}, \mathbf{1 3}, \mathbf{1 6}\}\{\mathbf{5}, \mathbf{7}, \mathbf{1 5}, \mathbf{1 6}\}\{\mathbf{1}, \mathbf{7}, \mathbf{1 1}, \mathbf{1 7}\}\{\mathbf{5}, \mathbf{1 0} \mathbf{1 3}, \mathbf{1 7}\} \\
\{\mathbf{6}, \mathbf{8}, \mathbf{1 5}, \mathbf{1 7}\}\{\mathbf{6}, \mathbf{9}, \mathbf{1 2}, \mathbf{1 6}\}\{\mathbf{3}, \mathbf{8}, \mathbf{1 0}, \mathbf{1 6}\}\{2,9,14,17\}\{1,9,10,18\}\{3,4,12,17\} \\
\{4,9,13,19\}\{4,11,15,18\}\{8,12,13,18\}\{2,6,7,18\}\{3,5,14,18\}\{1,5,8,19\} \\
\{7,12,14,19\}\{2,10,15,19\}\{3,6,11,19\}\{1,6,13,20\}\{4,7,10,20\}\{8,11,14,20\} \\
\{2,5,12,20\}\{3,9,15,20\}\{1,12,15,21\}\{6,10,14,21\}\{5,9,11,21\}\{2,4,8,21\} \\
\{3,7,13,21\}\end{array}$ \\
\hline $3^{6} 6^{1}$ & $\begin{array}{l}\{\mathbf{1 0}, \mathbf{1 3}, \mathbf{1 8}, \mathbf{2 0}\}\{\mathbf{3}, \mathbf{8}, \mathbf{1 1}, \mathbf{2 0}\}\{\mathbf{1}, \mathbf{7}, \mathbf{1 1}, \mathbf{1 6}\}\{\mathbf{3}, \mathbf{1 3}, \mathbf{1 6}, \mathbf{1 9}\}\{\mathbf{5}, \mathbf{8}, \mathbf{1 8}, \mathbf{1 9}\} \\
\{\mathbf{2}, \mathbf{5}, \mathbf{1 0}, \mathbf{1 4}\}\{\mathbf{6}, \mathbf{1 1}, \mathbf{1 4}, \mathbf{1 9}\}\{\mathbf{2}, \mathbf{4}, \mathbf{9}, \mathbf{1 9}\}\{\mathbf{1}, \mathbf{1 2}, \mathbf{1 7}, \mathbf{1 9}\}\{\mathbf{1}, \mathbf{5}, \mathbf{1 5}, \mathbf{2 0}\} \\
\{1,4,18,21\}\{7,14,18,22\}\{2,6,16,20\}\{4,8,13,17\}\{9,14,17,20\}\{4,7,12,20\} \\
\{7,10,15,19\}\{2,11,18,23\}\{3,5,7,21\}\{3,10,17,22\}\{3,4,14,23\}\{6,8,10,21\} \\
\{1,6,13,22\}\{6,7,17,23\}\{9,11,13,21\}\{5,9,16,22\}\{1,9,10,23\}\{12,14,16,21\} \\
\{2,8,12,22\}\{5,12,13,23\}\{2,15,17,21\}\{4,11,15,22\}\{8,15,16,23\}\{1,8,14,24\} \\
\{4,10,16,24\}\{5,11,17,24\}\{2,7,13,24\}\{3,6,9,12,15,18,24\}\end{array}$ \\
\hline $3^{10} 12^{1}$ & 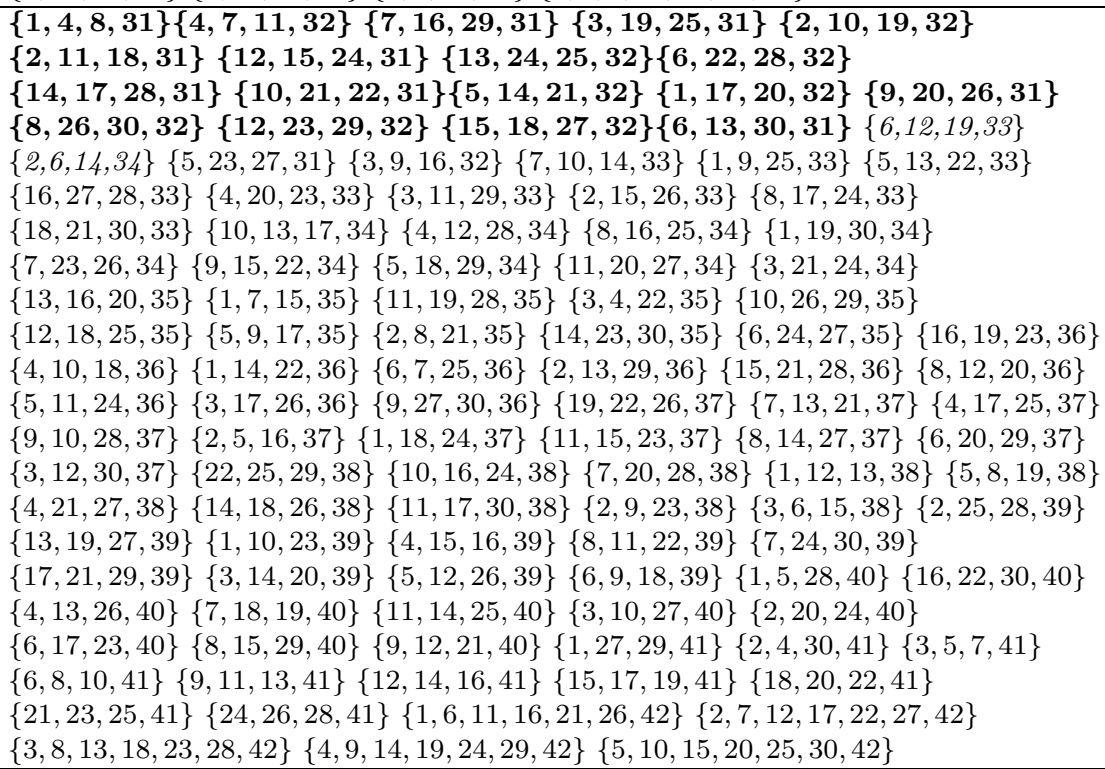 \\
\hline
\end{tabular}

\section{ACKNOWLEDGMENT}

The authors are grateful to Megan Dewar for making available her Ph.D. thesis [10] and to Jerzy Jaromczyk for making available the paper [15.

\section{REFERENCES}

1. A. Aggarwal and J. S. Vitter, The input/output complexity of sorting and related problems, Commun. ACM 31 (1988), no. 9, 1116-1127. MR.1021794 (90k:68029)

2. B. Alspach, K. Heinrich, and B. Mohar, A note on Hamilton cycles in block-intersection graphs, Finite geometries and combinatorial designs (Lincoln, NE, 1987), Contemp. Math., vol. 111, Amer. Math. Soc., Providence, RI, 1990, pp. 1-4. MR.1079733 (91h:05079)

3. J. R. Bitner, G. Ehrlich, and E. M. Reingold, Efficient generation of the binary reflected Gray code and its applications, Comm. ACM 19 (1976), no. 9, 517-521. MR0424386 (54:12349)

4. S. R. Blackburn and J. F. Mckee, Constructing k-radius sequences, arXiv:1006.5812v1 (2010).

5. A. E. Brouwer, A. Schrijver, and H. Hanani, Group divisible designs with block-size four, Discrete Math. 20 (1977), no. 1, 1-10. MR0465894 (57:5780) 
6. F. Chung, P. Diaconis, and R. Graham, Universal cycles for combinatorial structures, Discrete Math. 110 (1992), no. 1-3, 43-59. MR.1197444 (93m:05018)

7. C. J. Colbourn, D. G. Hoffman, and R. Rees, A new class of group divisible designs with block size three, J. Combin. Theory Ser. A 59 (1992), no. 1, 73-89. MR.1141323 (93f:05015)

8. C. J. Colbourn and A. Rosa, Triple Systems, Oxford Mathematical Monographs, Oxford University Press, New York, 1999. MR.1843379 (2002h:05024)

9. N. G. de Bruijn, A combinatorial problem, Nederl. Akad. Wetensch., Proc. 49 (1946), 758-764 = Indagationes Math. 8, 461-467 (1946). MR0018142 (8:247d)

10. M. Dewar, Gray codes, universal cycles and configuration orderings for block designs, Ph.D. thesis, Carleton University, Ottawa, Ontario, Canada, ProQuest LLC, Ann Arbor, MI, 2007. MR.2711021

11. P. Eades and B. McKay, An algorithm for generating subsets of fixed size with a strong minimal change property, Inform. Process. Lett. 19 (1984), no. 3, 131-133. MR782221 (86d:68058)

12. M. K. Fort, Jr. and G. A. Hedlund, Minimal coverings of pairs by triples, Pacific J. Math. 8 (1958), 709-719. MR0103831(21:2595)

13. S. P. Ghosh, Consecutive storage of relevant records with redundancy, Commun. ACM 18 (1975), no. 8, 464-471. MR0383863(52:4743)

14. J. W. Gilkerson and J. W. Jaromczyk, Restoring the order of images in a sequence of MRI slices, unpublished manuscript (2002).

15. J. W. Gilkerson, J. W. Jaromczyk, and Z. Lonc, On constructing sequences of radius $k$ using finite geometries, unpublished manuscript.

16. F. Gray, Pulse code communication, 1953 (file 1947), US Patent 2,632,058.

17. H.-D. O. F. Gronau, R. C. Mullin, and Ch. Pietsch, The closure of all subsets of $\{3,4, \cdots, 10\}$ which include 3, Ars Combin. 41 (1995), 129-161. MR1365159 (96m:05023)

18. D. R. Hare, Cycles in the block-intersection graph of pairwise balanced designs, Discrete Math. 137 (1995), no. 1-3, 211-221. MR1312454 (95m:05027)

19. P. Horák, D. A. Pike, and M. E. Raines, Hamilton cycles in block-intersection graphs of triple systems, J. Combin. Des. 7 (1999), no. 4, 243-246. MR.1691408 (2000b:05026)

20. P. Horák and A. Rosa, Decomposing Steiner triple systems into small configurations, Ars Combin. 26 (1988), 91-105. MR982111 (90a:05029)

21. G. Hurlbert, On universal cycles for $k$-subsets of an $n$-set, SIAM J. Discrete Math. 7 (1994), no. 4, 598-604. MR.1299088 (95k:05038)

22. B. Jackson, Universal cycles of 4-subsets and 5-subsets, unpublished manuscript.

23. B. W. Jackson, Universal cycles of $k$-subsets and $k$-permutations, Discrete Math. 117 (1993), no. 1-3, 141-150. MR1226137 (94d:05002)

24. J. W. Jaromczyk and Z. Lonc, Sequences of radius $k$ : how to fetch many huge objects into small memory for pairwise computations, Algorithms and Computation, Lecture Notes in Comput. Sci., vol. 3341, Springer, Berlin, 2004, pp. 594-605. MR2158364 (2006d:68048)

25. H. Lenz, Some remarks on pairwise balanced designs, Mitt. Math. Sem. Giessen (1984), no. 165, 49-62. MR.745869 (86g:05015)

26. F. Ruskey, Adjacent interchange generation of combinations, J. Algorithms 9 (1988), no. 2, 162-180. MR936104 (89d:68066)

27. C. Savage, A survey of combinatorial Gray codes, SIAM Rev. 39 (1997), no. 4, 605-629. MR 1491049 (98m:94052)

28. S. Sen, S. Chatterjee, and N. Dumir, Towards a theory of cache-efficient algorithms, J. Assoc. Comput. Mach. 49 (2002), no. 6, 828-858. MR2145858 (2005m:68271)

29. L. Storme, Finite geometry, The CRC Handbook of Combinatorial Designs (C. J. Colbourn and J. H. Dinitz, eds.), Chapman \& Hall, Boca Raton, FL, 2007, pp. 702-729. MR2246267 (2007i:05001)

30. R. M. Wilson, An existence theory for pairwise balanced designs. I. Composition theorems and morphisms, J. Combin. Theory Ser. A 13 (1972), 220-245. MR0304203 (46:3338) 
Division of Mathematical Sciences, School of Physical and Mathematical Sciences, Nanyang Technological University, Singapore

E-mail address: ymchee@ntu.edu.sg

Division of Mathematical Sciences, School of Physical and Mathematical Sciences, Nanyang Technological University, Singapore

E-mail address: lingsan@ntu.edu.sg

Division of Mathematical Sciences, School of Physical and Mathematical Sciences, Nanyang Technological University, Singapore

E-mail address: tanyin@ntu.edu.sg

Division of Mathematical Sciences, School of Physical and Mathematical Sciences, Nanyang Technological University, Singapore

E-mail address: ziandezhang@ntu.edu.sg 